\title{
A MATHEMATICAL PROGRAMMING METHOD FOR THE TOPOLOGY OPTIMIZATION OF A TRUSS-LIKE CONTINUUM
}

\author{
Hao Cui, Kemin Zhou \\ College of Civil Engineering, Huaqiao University, Xiamen, China \\ e-mail: zhoukm@hqu.edu.cn
}

\begin{abstract}
A mathematical programming method to optimize the distribution field of a truss-like material is presented. The densities and angles of members are optimized in two separate procedures in each iteration. An explicit sub-problem in a variable separation form is established at every iteration procedure. At each sub-problem, the stress constraint function is expanded into a trigonometric series of the member angles. According to the extreme condition, the optimal orientations of members are determined. The member densities are optimized using the method of moving asymptotes (MMA). Two examples demonstrate that the optimal truss-like structures are very close to analytic solutions.
\end{abstract}

Keywords: topology optimization, mathematical programming method, truss-like continuum

\section{Introduction}

Structural topology optimization has gained increasing attention in both theoretical research and practical applications in the recent decades due to the pioneering research of Prager and Rozvany (1977), Cheng and Olhoff (1981), Bendsøe and Kikuchi (1988) and Zhou and Rozvany (1991). Several optimization methods have been presented to date. Bendsøe and Kikuchi (1988) proposed a homogenization method, in which sizes and angles of microstructures in every element are optimized to present the optimal structural topology. To improve the efficiency of topology optimization, the method of the solid isotropic material with penalization (SIMP) was put forward (Bendsøe, 1989; Rozvany et al., 1992). Xie and Steven (1993) presented an evolutionary structural optimization (ESO) method by deleting inefficient elements to form holes in the uniform isotropic continuum. Wang et al. (2003) proposed the level set method to optimize structural topology by optimizing the level set function which represents the boundary lines of the continuum. These methods have been widely applied to the numerical solution of topology optimization problems and have received considerable attention (Eschenauer and Olhoff, 2001; Bendsøe et al., 2005; Guo and Cheng, 2010; Baratta and Corbi, 2014). For more details, the readers are referred to the relevant literature and the references therein for recent advances in this area (Guo and Cheng, 2010; Sigmund and Maute, 2013; Deaton and Grandhi, 2014). In addition, new advances have been made in topology optimization methods in past several years. The moving morphable component (MMC) approach (Guo et al., 2014a) and the moving morphable voids (MMV) approach (Zhang et al., 2017) were put forward successively, in which topology optimization can be achieved in an explicit and geometrical way. In the MMC approach, a set of moving morphable components are used as the building blocks of topology optimization, while a set of moving morphable voids are employed to optimize the structural topology in the MMV approach. However, most research on the structural topology optimization primarily focus on a uniform isotropic continuum. The optimal structural topology is presented by the void and solid material or by the boundaries of the holes in the uniform isotropic continuum. All optimal structures mentioned above are uniform isotropic continua with holes. 
Michell (1904) revealed that topology optimization structures are generally non-uniform anisotropic truss-like continua in theory. The uniform isotropic continua with holes optimized by various numerical optimization methods are a kind of approximation of non-uniform anisotropic continua under the consideration for engineering application. Zhou and Li (2005) presented a truss-like material model. Truss-like material models can describe topology optimization structures more precisely. There are clear relations between the truss-like continuum and members. Therefore, the truss-like continuum can be transferred to frame easily. There are clear force transfer paths in the truss-like continuum. The remaining parts of members in this truss-like continuum can form a frame or a uniform isotropic continuum with holes. Therefore, topology optimization of the truss-like continuum is valuable.

At present, the structural topology optimization focuses mainly on global structural performances such as the structural compliance and natural frequency. However, local structural performances play an important role in designing industrial products and engineering structures. Among them, the local stress magnitude is a key point that must be taken into consideration to guarantee the strength of a product or a structure. However, topology optimization with stress constraints is more complicated than global structural performance-oriented topology optimization problems. Since a stress constraint is a local constraint, when stress constraints are considered, many constraint equations must be introduced into the optimization problem, which increases the difficulty of solving it. Duysinx and Bendsøe (1998) performed pioneering research on topology optimization with stress constraints under the SIMP framework. This is the basis on which topology optimization with stress constraints has been widely studied under the SIMP framework (Pereira et al., 2004; Bruggi, 2008; Bruggi and Venini, 2008; París et al., 2009; Le et al., 2010; Holmberg et al., 2013; Kiyono et al., 2016). In addition to SIMP-based methods, numerous level set-based methods have also been proposed to solve topology optimization problems with stress constraints (Allaire and Jouve, 2008; Guo et al., 2011, 2014b; Zhang et al., 2013; Wang and Li, 2013; Xia et al., 2012; Picelli et al., 2018). It is also worth highlighting that topology optimization problems with stress constraints can also be solved using a reaction-diffusion equation (Emmendoerfer and Fancello, 2016), topological derivative information (Amstutz and Novotny, 2010; Suresh and Takalloozadeh, 2013), constraint aggregation method (Kennedy and Hicken, 2015) and finite cell method (Parvizian et al., 2012; Cai et al., 2014; Cai and Zhang, 2015).

Although a series of achievements have been made for solving topology optimization problems with stress constraints, there is still considerable room for further improvement of the methods mentioned above. For example, in terms of stress-constrained topology optimization using SIMP-based approaches, one difficulty is the "stress singularity phenomenon". Some technological measures must be taken to tackle it appropriately. In topology optimization of truss-like with stress constraints, the stress ratio method of the fully stressed criterion is generally adopted. The densities and orientations of members are adjusted according to the magnitude and orientation of the principal stress (Zhou and Li, 2005). The optimization method based on the fully stressed criterion has high efficiency and is not sensitive to the number of design variables. However, the stress ratio method of the fully stressed criterion has fatal limitations, which only applies to the topology optimization problem with stress constraints under the single load case. Although the mathematical programming method requires long times and more computer memory for computing, it has broad prospects with the development of computer science. Furthermore, the mathematical programming method has a solid theoretical foundation and adaptability to complex problems. It can be used to solve multi-constraint optimization problems, such as constraints on stresses, displacements, natural frequency and buckling instability of the structure. Mathematical programming methods, including sequential linear programming (SLP), sequential quadratic programming (SQP), and the method of moving asymptotes (MMA) (Svanberg, 1987), 
expand the objective function and constraint function as polynomials in separated variable forms. These methods are applied widely in structural topology optimization.

In this paper, a mathematic programming method to optimize the distribution field of a truss-like material is presented. The truss-like material model of two planar families of orthotropic members is adopted. The densities and directions of the two families of members at nodes are taken as design variables. The structural material volume is the objective function. The densities and orientations of members are optimized respectively in every iteration. The stress constraints are expanded as trigonometric functions in a separate variable form with respect to the member angles, approximately. The method of moving asymptotes is adopted to optimize the member densities. No numerical instability exists in optimization iterations, and the method has a strong adaptability to multi-constraint optimization problems.

\section{Truss-like material model and FEM}

\subsection{Truss-like continuum material model and elastic matrix}

It is assumed that, in the truss-like continuum, non-uniformed dense members are continuously distributed in the design domain. According to the feature of the optimization problem, different material models are presented such as planar two families of orthotropic members (Zhou and Li, 2006), planar three families of non-orthotropic members (Zhou and Li, 2011) or spatial three families of orthotropic members (Zhou and Li, 2005). In this paper, to optimize the least-weight planar structure with stress constraints under the single load case, the planar truss-like material model with two families of orthotropic members is adopted. The densities and the orientation angle of two families of members are denoted by $t_{1}, t_{2}$ and $\alpha$, respectively. The elastic matrix is assumed as follows (Zhou and Li, 2006)

$$
\mathbf{D}\left(t_{1}, t_{2}, \alpha\right)=E \sum_{b=1}^{2} t_{b} \sum_{r=1}^{3} s_{b r} g_{r}(\alpha) \mathbf{A}_{r}
$$

where $s_{b r}$ and $g_{r}$ are components of the constant matrix $\mathbf{s}$ and the functional vector $\mathbf{g}$

$$
\mathbf{s}=\left[\begin{array}{ccc}
1 & 1 & 1 \\
-1 & -1 & 1
\end{array}\right] \quad \mathbf{g}(\alpha)=[\cos 2 \alpha, \sin 2 \alpha, 1]
$$

respectively, with $\mathbf{A}_{r}$ being the constant matrix, and $E$ - Young's modulus

$$
\mathbf{A}_{1}=\frac{1}{2} \operatorname{diag}\left[\begin{array}{lll}
1 & -1 & 0
\end{array}\right] \quad \mathbf{A}_{2}=\frac{1}{4}\left[\begin{array}{lll}
0 & 0 & 1 \\
0 & 0 & 1 \\
1 & 1 & 0
\end{array}\right] \quad \mathbf{A}_{3}=\frac{1}{2} \operatorname{diag}\left[\begin{array}{lll}
1 & 1 & \frac{1}{2}
\end{array}\right]
$$

The structure is analysed using the finite element method. The densities $t_{1 j}, t_{2 j}$ and the orientation angle $\alpha_{j}$ of members at nodes $j(j \in J)$ are taken as design variables. $J$ is the set of all nodes in the design domain. The elastic matrix at any point within an element $e$ is calculated by interpolation of the elastic matrices at nodes belonging to the element $e$ using the shape function $N_{j}(\xi, \eta)$ as follows

$$
\mathbf{D}_{e}(\xi, \eta)=\sum_{j \in S_{e}} N_{j}(\xi, \eta) \mathbf{D}\left(t_{1 j}, t_{2 j}, \alpha_{j}\right)
$$

where $\xi, \eta$ are the local coordinates in an element, and $S_{e}$ is the set of nodes belonging to element $e$. Introducing Eq. (2.1) into Eq. (2.4) leads to the elastic matrix at any point within the element

$$
\mathbf{D}_{e}=E \sum_{j \in S_{e}} N_{j} \sum_{b=1}^{2} t_{b j} \sum_{r=1}^{3} s_{b r} g_{r}\left(\alpha_{j}\right) \mathbf{A}_{r}
$$




\subsection{Stiffness matrix of truss-like continuum}

The element stiffness matrix is calculated by the following integration according to the basic theory of the finite element method as follows

$$
\mathbf{k}_{e}=\int_{V_{e}} \mathbf{B}^{\mathrm{T}} \mathbf{D}_{e} \mathbf{B} d V
$$

where $\mathbf{B}$ is the geometry matrix, and $V_{e}$ is the volume of the element $e$. Introducing Eq. (2.5) into Eq. (2.6) leads to the following

$$
\mathbf{k}_{e}=E \sum_{j \in S_{e}} \sum_{b=1}^{2} t_{b j} \sum_{r=1}^{3} s_{b r} g_{r}\left(\alpha_{j}\right) \int_{V_{e}} N_{j} \mathbf{B}^{\mathrm{T}} \mathbf{A}_{r} \mathbf{B} d V=\sum_{j \in S_{e}} \sum_{b=1}^{2} t_{b j} \sum_{r=1}^{3} s_{b r} g_{r}\left(\alpha_{j}\right) \mathbf{H}_{e j r}
$$

where

$$
\mathbf{H}_{e j r}=E \int_{V_{e}} N_{j} \mathbf{B}^{\mathrm{T}} \mathbf{A}_{r} \mathbf{B} d V
$$

is a constant matrix which is independent of design variables. If regular elements are adopted, $H_{\text {ejr }}$, being independent of the elements, can be calculated before optimization.

The structural stiffness matrix can be obtained by summation of all element stiffness matrices as follows

$$
\mathbf{K}=\sum_{e} \mathbf{k}_{e}=\sum_{e} \sum_{j \in S_{e}} \sum_{b=1}^{2} t_{b j} \sum_{r=1}^{3} s_{b r} g_{r}\left(\alpha_{j}\right) \mathbf{H}_{e j r}=\sum_{j \in J} \sum_{e \in S_{j}} \sum_{b=1}^{2} t_{b j} \sum_{r=1}^{3} s_{b r} g_{r}\left(\alpha_{j}\right) \mathbf{H}_{e j r}
$$

where the first summation notation represents the assembling structural stiffness matrix from the elementary stiffness matrix, and $S_{j}$ is the set of elements around the node $j$. The exchange of accumulation order leads to the second equation, which is convenient for following derivation.

To optimize the structures using a mathematical programming method, the sensitivities of structural stiffness with respect to design variables are calculated by the partial derivative of Eq. (2.9) as follows

$$
\frac{\partial \mathbf{K}}{\partial t_{b j}}=\sum_{e \in S_{j}} \sum_{r=1}^{3} s_{b r} g_{r}\left(\alpha_{j}\right) \mathbf{H}_{e j r} \quad \frac{\partial \mathbf{K}}{\partial \alpha_{j}}=\sum_{e \in S_{j}} t_{b j} \sum_{r=1}^{2} s_{b r} g_{r}^{\prime}\left(\alpha_{j}\right) \mathbf{H}_{e j r}
$$

where $g_{r}^{\prime}\left(\alpha_{j}\right)$ is the derivative of the second equation $g_{r}\left(\alpha_{j}\right)$ of Eq. (2.2) with respect to the member angle $\alpha_{j}$

$$
\mathbf{g}^{\prime}\left(\alpha_{j}\right)=2\left[-\sin 2 \alpha_{j}, \cos 2 \alpha_{j}, 0\right]
$$

\subsection{Finite element analyses}

By solving the equilibrium equation

$$
\mathbf{K U}=\mathbf{F}
$$

where $\mathbf{F}$ and $\mathbf{U}$ are the load and nodal displacement vector, respectively, the nodal displacement vector $\mathbf{U}$ can be obtained from the following

$$
\mathbf{U}=\mathbf{K}^{-1} \mathbf{F}
$$


The sensitivity of nodal displacement can be calculated via Eq (2.12) as follows

$$
\frac{\partial \mathbf{U}}{\partial t_{b j}}=-\mathbf{K}^{-1} \frac{\partial \mathbf{K}}{\partial t_{b j}} \mathbf{U} \quad \frac{\partial \mathbf{U}}{\partial \alpha_{j}}=-\mathbf{K}^{-1} \frac{\partial \mathbf{K}}{\partial \alpha_{j}} \mathbf{U}
$$

Strain $\varepsilon_{j}=\left[\varepsilon_{x}, \varepsilon_{y}, \gamma_{x y}\right]_{j}^{\mathrm{T}}$ at the node $j$ is calculated by the average of strains at the nodes belonging to the elements near the node $j$

$$
\varepsilon_{j}=\frac{1}{n_{j}} \sum_{e \in S_{j}} \mathbf{B}_{j} \mathbf{U}_{e}
$$

where $\mathbf{B}_{j}$ is the geometry matrix of elements around the node $j, n_{j}$ is the number of elements near node $j$, and $\mathbf{U}_{e}$ is the nodal displacement vector of the element. For convenience, Eq. (2.15) is denoted simply as follows

$$
\varepsilon_{j}=\mathbf{B}_{j} \mathbf{U}_{e}
$$

The strains of two members at the node $j$ are calculated by the following

$$
\varepsilon_{b j}=\mathbf{T}_{b j} \varepsilon_{j}=\mathbf{T}_{b j} \mathbf{B}_{j} \mathbf{U}_{e}
$$

where

$$
\begin{aligned}
& \mathbf{T}_{1 j}=\frac{1}{2}\left[1+\cos 2 \alpha_{j}, 1-\cos 2 \alpha_{j}, \sin 2 \alpha_{j}\right] \\
& \mathbf{T}_{2 j}=\frac{1}{2}\left[1-\cos 2 \alpha_{j}, 1+\cos 2 \alpha_{j},-\sin 2 \alpha_{j}\right]
\end{aligned}
$$

The member strain denoted by Eq. (2.17) is differentiated with respect to $\alpha_{i}(i \in J)$

$$
\frac{\partial \varepsilon_{b j}}{\partial \alpha_{i}}=\mathbf{T}_{b j}^{\prime} \mathbf{B}_{j} \mathbf{U}_{e} \delta_{i j}+\mathbf{T}_{b j} \mathbf{B}_{j} \frac{\partial \mathbf{U}_{e}}{\partial \alpha_{i}} \quad \frac{\partial^{2} \varepsilon_{b j}}{\partial \alpha_{i}^{2}}=\mathbf{T}_{b j}^{\prime \prime} \mathbf{B}_{j} \mathbf{U}_{e} \delta_{i j}+\mathbf{T}_{b j} \mathbf{B}_{j} \frac{\partial^{2} \mathbf{U}_{e}}{\partial \alpha_{i}^{2}}
$$

where

$$
\begin{array}{lc}
\mathbf{T}_{1 j}^{\prime}=\left[-\sin 2 \alpha_{j}, \sin 2 \alpha_{j}, \cos 2 \alpha_{j}\right] & \mathbf{T}_{2 j}^{\prime}=-\mathbf{T}_{1 j}^{\prime} \\
\mathbf{T}_{2 j}^{\prime \prime}=2\left[-\cos 2 \alpha_{j}, \cos 2 \alpha_{j},-\sin 2 \alpha_{j}\right] & \mathbf{T}_{2 j}^{\prime \prime}=-\mathbf{T}_{1 j}^{\prime \prime}
\end{array}
$$

and $\delta_{i j}$ is the Kronecker delta.

\subsection{Material volume of truss-like continuum}

The densities of the member at nodes are taken as design variables, and the densities of members at any point within an element can be calculated by interpolation of the member densities at nodes belonging to the element with the aid of the shape function $N_{j}(\xi, \eta)$

$$
t_{b}(\xi, \eta)=\sum_{j \in S_{e}} N_{j}(\xi, \eta) t_{b j}
$$

The structural material volume is calculated by integration of the member over all elements

$$
V=\sum_{e} \sum_{b} \int_{V_{e}} \sum_{j \in S_{e}} N_{j} t_{b j} d V=\sum_{j \in J} \sum_{e \in S_{j}} \int_{V_{e}} N_{j} d V \sum_{b} t_{b j}=\sum_{j \in J} z_{j} \sum_{b} t_{b j}
$$

where

$$
z_{j}=\sum_{e \in S_{j}} \int_{V_{e}} N_{j} d V
$$

The sensitivities of the material volume (objective function) with respect to the design variables are expressed as follows

$$
\frac{\partial V}{\partial t_{b j}}=z_{j} \quad \frac{\partial V}{\partial \alpha_{j}}=0
$$




\section{Optimization method of truss-like continuum}

The optimization problem of the least-weight structure with stress constraints is expressed as follows:

$$
\left\{\begin{array}{lll}
\text { find } & t_{b j}, \alpha_{j} & b=1,2 \\
\min & V & j \in J \\
\text { s.t. } & E\left|\varepsilon_{b j}\right| \leqslant \sigma_{p} &
\end{array}\right.
$$

where $\sigma_{p}$ is the allowable stress. In each iteration, the densities and the angle of the member are optimized in two independent procedures separately.

\subsection{Optimization of member densities}

The strain of members at every node are expanded according to the Method of Moving Asymptotes (MMA) (Svanberg, 1987) as follows

$$
\varepsilon_{b j}=\varepsilon_{b j}^{(k)}+\sum_{i \in J} \sum_{a=1}^{2}\left(\frac{p_{b j a i}}{U_{a i}-t_{a i}}-\frac{p_{b j a i}}{U_{a i}-t_{a i}^{(k)}}+\frac{q_{b j a i}}{t_{a i}-L_{a i}}-\frac{q_{b j a i}}{t_{a i}^{(k)}-L_{a i}}\right) \quad i, j \in J
$$

where

$$
\frac{\partial \varepsilon_{b j}}{\partial t_{a i}}=\mathbf{T}_{b j} \mathbf{B}_{j} \frac{\partial \mathbf{U}_{e}}{\partial t_{a i}}
$$

and

$$
p_{b j a i}=\max \left[0,\left(U_{b j}-t_{b j}^{(k)}\right)^{2} \frac{\partial \varepsilon_{b j}}{\partial t_{a i}}\right] \quad q_{b j a i}=\max \left[0,-\left(t_{b j}^{(k)}-L_{b j}\right)^{2} \frac{\partial \varepsilon_{b j}}{\partial t_{a i}}\right]
$$

$k$ is the iterative index. The member densities are optimized by MMA.

\subsection{Member strain expanded into trigonometric function}

From Eq. (2.24), we find that the objective function (structural material volume) is independent of member angles. Only the constraint functions (member strain) depend on the member angles, and the angle variables are unbounded. Therefore, in optimization problem (3.1), the angle variables are distinctly different from the density variables. For this reason, the angles and densities of the member are optimized separately in two independent procedures in each iteration. To find the optimal angles, we should minimize the absolute value of the member strain.

To minimize the member strain, the sensitivities of members about the angle are needed. The sensitivities of the member strain with respect to the member angle are intended to be expanded into a Fourier series. As the member strain is a multi-variable function, whose independent variables are member orientation angles at all nodes in the design domain, it is thus a multivariate Fourier series. Therefore, it is hard to solve the optimization problem directly. To find the optimal orientation angles according to an extreme condition, we must introduce some assumptions to simplify the multivariate Fourier series. Thus, the iterative idea of the mathematics of computation can be used to find the optimal orientation angles.

It is known that a change in the member angle at the node $j$ will induce changes in the stiffness matrix, which results in changes in the displacement field. However, a change in the displacement near the node $j$ will be larger greatly than that at other nodes far from the node $j$. For the sake of simplicity, the influence of the member angle only at the node $j$ on the member strain at the node $j$ is taken into consideration. Therefore, the member strain at the node $j$ is 
approximately treated as a function of the member orientation angle at the node $j$. The angle of the member at every node can be optimized independently in each iteration. In addition, the effect of the angle change on the displacement field is omitted, namely, taking $\partial \mathbf{U}_{e} / \partial \alpha_{j}$ outside the integral. Thus, the sensitivity of the member strain at the node $j$ with respect to the member angle at the node $j$ is expanded as follows

$$
\frac{\partial \varepsilon_{b j}}{\partial \alpha_{j}} \doteq c_{0 b j}^{1}+\sum_{n=1}^{\infty}\left(c_{n b j}^{1} \cos 2 n \alpha_{j}+d_{n b j}^{1} \sin 2 n \alpha_{j}\right)
$$

and

$$
\begin{aligned}
& c_{0 b j}^{1}=\frac{1}{\pi} \int_{0}^{\pi} \frac{\partial \mathbf{T}_{b j}}{\partial \alpha_{j}} d \alpha_{j} \mathbf{B}_{j} \mathbf{U}_{e}+\frac{1}{\pi} \int_{0}^{\pi} \mathbf{T}_{b j} d \alpha_{j} \mathbf{B}_{j} \frac{\partial \mathbf{U}_{e}}{\partial \alpha_{j}} \\
& c_{n b j}^{1} \doteq \frac{2}{\pi} \int_{0}^{\pi} \frac{\partial \mathbf{T}_{b j}}{\partial \alpha_{j}} \cos 2 n \alpha_{j} d \alpha_{j} \mathbf{B}_{j} \mathbf{U}_{e}+\frac{2}{\pi} \int_{0}^{\pi} \mathbf{T}_{b j} \cos 2 n \alpha_{j} d \alpha_{j} \mathbf{B}_{j} \frac{\partial \mathbf{U}_{e}}{\partial \alpha_{j}} \\
& d_{n b j}^{1} \doteq \frac{2}{\pi} \int_{0}^{\pi} \frac{\partial \mathbf{T}_{b j}}{\partial \alpha_{j}} \mathbf{B}_{j} \mathbf{U}_{e} \sin 2 n \alpha_{j} d \alpha_{j}+\frac{2}{\pi} \int_{0}^{\pi} \mathbf{T}_{b j} \sin 2 n \alpha_{j} d \alpha_{j} \mathbf{B}_{j} \frac{\partial \mathbf{U}_{e}}{\partial \alpha_{j}} \quad(n=1,2,3, \ldots)
\end{aligned}
$$

The period of the member angle is $\pi$ because it has identical orientation if the member angle is increased by $\pi$. Therefore, the variables in the Fourier expansion are double angles.

The first term on the right-hand side of Eq. (3.6) denotes the change of the member strain induced directly by the change of the member angle under the prescribed displacement field; the second term on the right-hand side of Eq. (3.6) denotes the change of the member strain induced by the change of the displacement field.

Introducing the first equation of Eq. (2.18) and Eq. (2.20) into Eq. (2.19) $)_{1}$ leads to the following

$$
\frac{\partial \varepsilon_{b j}}{\partial \alpha_{j}}=\left[-\sin 2 \alpha_{j}, \sin 2 \alpha_{j}, \cos 2 \alpha_{j}\right] \mathbf{B}_{j} \mathbf{U}_{e}+\frac{1}{2}\left[1+\cos 2 \alpha_{j}, 1-\cos 2 \alpha_{j}, \sin 2 \alpha_{j}\right] \mathbf{B}_{j} \frac{\partial \mathbf{U}_{e}}{\partial \alpha_{j}}
$$

Here, only the first three terms remain in trigonometric series (3.5) for simplicity. Comparing Eqs. (3.6) with Eq. $(2.19)_{1}$, it is easy to find that

$$
\left[\begin{array}{l}
c_{0 b j}^{1} \\
c_{1 b j}^{1} \\
d_{1 b j}^{1}
\end{array}\right]=2\left[\begin{array}{ccc}
0 & 0 & 0 \\
0 & 0 & 1 \\
-1 & 1 & 0
\end{array}\right] \mathbf{B}_{j} \mathbf{U}_{e}+\left[\begin{array}{ccc}
1 & 1 & 0 \\
1 & -1 & 0 \\
0 & 0 & 1
\end{array}\right] \mathbf{B}_{j} \frac{\partial \mathbf{U}_{e}}{\partial \alpha_{j}}
$$

With the definition of symbols

$$
\lambda_{b j}=\sqrt{\left(c_{1 b j}^{1}\right)^{2}+\left(d_{1 b j}^{1}\right)^{2}} \quad \theta_{j}=\frac{1}{2} \arctan \frac{d_{1 b j}^{1}}{c_{1 b j}^{1}}
$$

namely

$$
\cos 2 \theta_{j}=\frac{c_{1 b j}^{1}}{\lambda_{b j}} \quad \sin 2 \theta_{j}=\frac{d_{1 b j}^{1}}{\lambda_{b j}}
$$

Equation (3.5) can be expressed as follows

$$
\frac{\partial \varepsilon_{b j}}{\partial \alpha_{j}}=\frac{1}{2}\left[c_{0 b j}^{1}+\lambda_{b j} \cos 2\left(\theta_{j}-\alpha_{j}\right)\right]
$$


To obtain the extremum of the member strain $\varepsilon_{b j}$, Eq. (3.11) should be equal to zero, and thus

$$
\theta_{j}-\alpha_{j}= \pm \frac{1}{2} \arccos \left(-\frac{c_{0 b j}^{1}}{\lambda_{b j}}\right)
$$

Further, we take the derivative of Eq. (3.11) with respect to the angle $\alpha_{j}$ as follows

$$
\frac{\partial^{2} \varepsilon_{1 j}}{\partial \alpha_{j}^{2}}=\lambda_{b j} \sin 2\left(\theta_{j}-\alpha_{j}\right)
$$

To minimize the member strain, Eq. (3.13) should be greater than or equal to zero

$$
\frac{\partial^{2} \varepsilon_{1 j}}{\partial \alpha_{j}^{2}}=\lambda_{b j} \sin 2\left(\theta_{j}-\alpha_{j}\right) \geqslant 0
$$

From Eq (3.14), we get the following

$$
0 \leqslant \theta_{j}-\alpha_{j} \leqslant \frac{\pi}{2}
$$

Therefore, to minimize the member strain, the plus sign in Eq. (3.12) should remain as follows

$$
\theta_{j}-\alpha_{j}=\frac{1}{2} \arccos \left(-\frac{c_{0 b j}^{1}}{\lambda_{b j}}\right)
$$

Otherwise, to obtain the maximum member strain, the minus sign in Eq. (3.12) should remain as follows

$$
\theta_{j}-\alpha_{j}=-\frac{1}{2} \arccos \left(-\frac{c_{0 b j}^{1}}{\lambda_{b j}}\right)
$$

In the discussion above, only the principal values of the inverse trigonometric function are taken into consideration, because the orientation of the member is identical when the member angle is increased by $\pi$.

\subsection{Optimization method of member angles}

As mentioned above, to find the optimal angles, we should minimize the absolute value of the member strain as follows

$$
\min _{\alpha_{j}, j \in J}\left|\varepsilon_{b j}\right|
$$

The member strains can be expanded to trigonometric functions into the separate variables form. The angle of the member at every node can be optimized independently.

The absolute value operator will make the constraint functions (member strain) unsmooth. However, this difficulty can be overcome easily, since the strain at the extreme point is close to the permitted strain, which is far from zero. This means that the sign will not change near the optimal point. Thus, whether the strain of the member achieves a minimum or a maximum can be determined according to its sign

$$
\left\{\begin{array}{lll}
\min _{\alpha_{j}, j \in J} \varepsilon_{b j} & \text { if } & \varepsilon_{b j}>0 \\
\max _{\alpha_{j}, j \in J} \varepsilon_{b j} & \text { if } & \varepsilon_{b j}<0
\end{array}\right.
$$

In the case of $\left|c_{0 b j}^{1}\right|>\lambda_{b j}$, the solution to Eq. (3.11) does not exist. Otherwise, it is true that $\left|c_{0 b j}^{1}\right| \leqslant \lambda_{b j}$. Equations (3.16) and (3.17) are rewritten as follows

$$
\alpha_{j}=\left\{\begin{array}{ccc}
\theta_{j}-\frac{1}{2} \arccos \left(-\frac{c_{0 b j}^{1}}{\lambda_{b j}}\right) & \text { if } \quad \varepsilon_{b j} \geqslant 0 \\
\theta_{j}+\frac{1}{2} \arccos \left(-\frac{c_{0 b j}^{1}}{\lambda_{b j}}\right) & \text { if } \quad \varepsilon_{b j}<0
\end{array}\right.
$$




\subsection{Optimization procedure}

The densities and angles of the member at nodes are taken as design variables. The densities and the angle of the member are optimized separately in each iteration. The objective function and the constraint function are expanded into an explicit convex function with respect to member densities, according to the method of moving asymptotes (MMA), which is easy to solve (Svanberg, 1987). The member angles are optimized according to Eq. (3.20) derived above. The concrete optimization procedure is as follows:

(1) The design domain is meshed by finite elements. Design variables are initialized;

(2) The structure is analysed by the finite element method;

(3) Sensitivities of the objective function and the constraint function are analysed;

(4) The objective and constraint functions about densities are expanded into a separate variables form. The member densities are optimized using the method of moving asymptotes;

(5) The constraint function about angle is expanded as in Eq. (3.11). The member angles are updated according to Eq. (3.20);

(6) Return to step (2) if the change in the objective and constraint functions excess $1 \%$. Otherwise, the iterations are terminated. The optimal truss-like continua are obtained;

(7) Optimal truss-like continua are illustrated.

\subsection{Demonstration of optimal truss-like continuum}

A truss-like structure is a type of non-uniform anisotropic continuum. Members in the truss-like structure are distributed continuously. To demonstrate the truss-like structure, two types of figures are adopted with crossed lines and continuous lines, respectively. Using the crossed lines, the densities and angles of members are presented using two short lines at every node. The angles and lengths of the two lines stand for the angles and densities of two families of members at every node. A few lines that are too long are cut short to make the figure distinguishable.

Another figure to show the truss-like structure is a series of continuous lines. There are two types of continuous lines in the figure which are drawn as in the following procedure. Firstly, one type of lines are drawn from the nodes with larger member densities (including supports, points acted by point forces, etc.) to the elementary boundary. The angle at the point at which the line intersects the elementary boundary is calculated by interpolation of the angles at the two end nodes of the elementary boundary, which is taken as the angle of the next segment line in the neighbour element. The next segment line is drawn in the neighbour element along the angle calculated just above. This procedure is continuously repeated from one element to another until the line reaches the design domain boundary, which creates a broken line. The broken line, consisting of many segments of straight lines, stands for a curve approximately. Secondly, not being the main path for force transfer, the other type of lines is drawn from the points equidistantly distributed on the curve just drawn above in its orthotropic orientations. Since the members are distributed continuously in the truss-like structure, only parts of the members are remained. The quantity of the second type of lines can be determined by the actual needs of the practical engineering.

\section{Numerical examples}

The classical Michell trusses are taken as two numerical examples. Young's modulus is $E=210 \mathrm{GPa}$. The permitted stress is $\sigma_{p}=160 \mathrm{MPa}$. The point force acted at structure is $F=10^{5} \mathrm{kN}$. Four-node rectangular elements are adopted. 


\subsection{Example 1}

A short cantilever fixed at the left boundary is acted on by a point force downward at the middle of the right boundary, as shown in Fig. 1a. $16 \times 10$ elements are used. The truss-like material distribution field is optimized after 8 iterations. The densities and orientations of the members at nodes are expressed by lengths and orientations of lines, respectively, as shown in Fig. 1b. To demonstrate the optimal structure, a series of continuous lines are drawn in many elements according to the densities and orientation at elemental boundaries programmatically, as shown in Fig. 1c. For comparison, the analytical solution (Hemp, 1973) is shown in Fig. 1d.

(a)

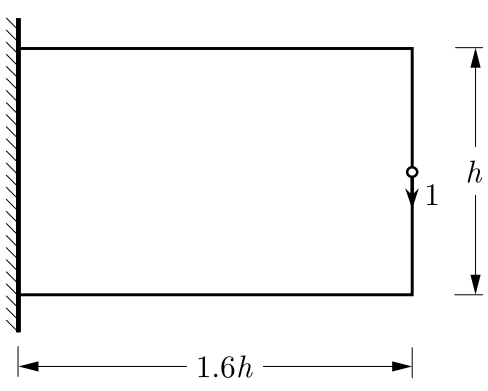

(c)

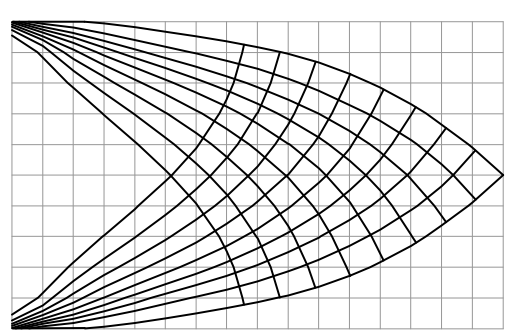

(b)

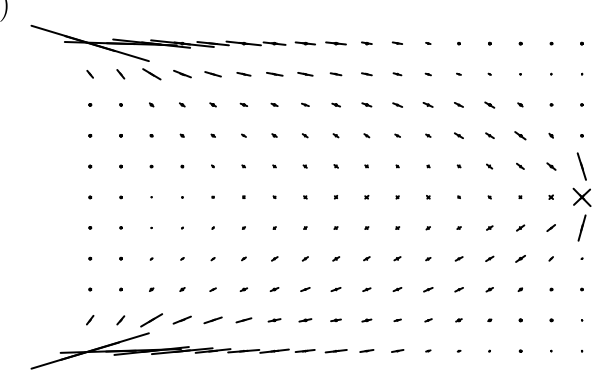

(d)

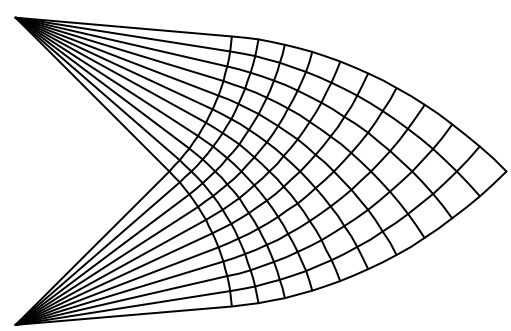

Fig. 1. Mechanical model and analysis results of example 1; (a) mechanical model, (b) optimal truss-like continuum, (c) optimal truss-like continuum presented by continuous lines, (d) analytical solution

\subsection{Example 2}

A simply supported beam is acted on by a point force downward at the centre of the bottom, as shown in Fig. 2a. $20 \times 12$ elements are used. The truss-like material distribution field is optimized after 10 iterations. Figures $2 \mathrm{~b}$ and $2 \mathrm{c}$ demonstrate the optimal truss-like structure by crossed lines and continuum lines, respectively. For comparison, the analytical solution (Hemp, 1973) is shown in Fig. 2d.

From the two numerical examples, it is found that the optimal truss-like continua are very close to the analytical solution with less finite elements. There are no numerical instabilities.

\section{Conclusions}

A mathematical programming method to optimize the distribution field of a truss-like material is presented. The densities and angles of members are optimized in two separate procedures in each iteration. The stress constraints are approximately expanded into trigonometric functions in a separate variable form with respect to member angles. The method of moving asymptotes is adopted to optimize the member densities.

Although the results only provide an optimal material distribution field, rather than definite structures represented by a solid material or a void, it provides more flexibility for the designer to choose appropriate concrete structures under consideration for practical demands of engineering. 
(a)

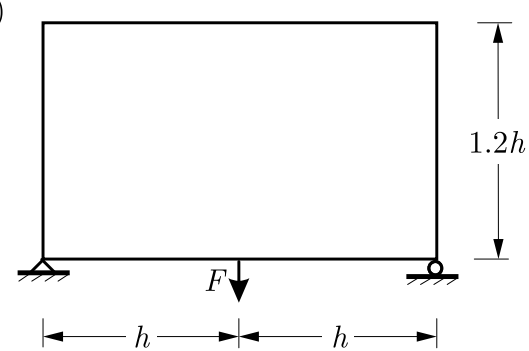

(c)

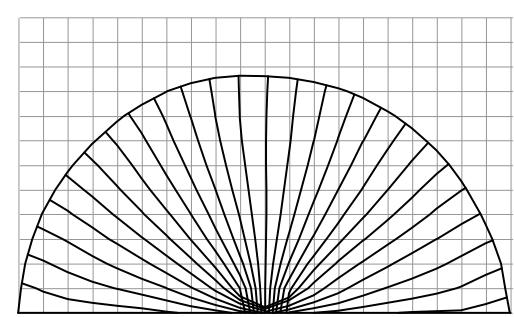

(b)

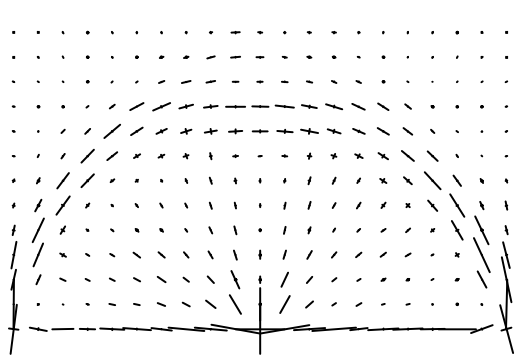

(d)

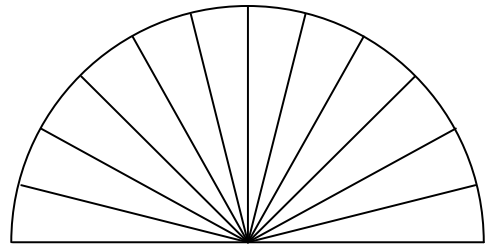

Fig. 2. Mechanical model and analysis results of example 2; (a) mechanical model, (b) optimal truss-like continuum, (c) optimal truss-like continuum presented by continuous lines, (d) analytical solution

Based on the optimal truss-like structures, uniform isotropic perforated plates and discrete trusses can be achieved.

\section{Acknowledgements}

This work was financially supported by the National Natural Science Foundation of China (No. 11572131) and the Subsidized Project for Postgraduates' Innovative Fund in Scientific Research of Huaqiao University (No. 17011086002).

\section{References}

1. Allaire G., Jouve, F., 2008, Minimum stress optimal design with the level set method, Engineering Analysis with Boundary Elements, 32, 11, 909-918

2. Amstutz S., Novotny A.A., 2010, Topological optimization of structures subject to von Mises stress constraints, Structural and Multidisciplinary Optimization, 41, 3, 407-420

3. Baratta A., Corbi I., 2014, Topology optimization for reinforcement of no-tension structures, Acta Mechanica, 225, 3, 663-678

4. Bendsøe M.P., 1989, Optimal shape design as a material distribution problem, Structural Optimization, 1, 4, 193-202

5. Bendsøe M.P., Kikuchi E., 1988, Generating optimal topologies in structural design using a homogenization method, Computer Methods in Applied Mechanics and Engineering, 71, 2, 197-224

6. Bendsøe M.P., Lund E., Olhoff N., Sigmund O., 2005, Topology optimization broadening the areas of application, Control and Cybernetics, 34, 1, 7-35

7. Bruggi M., 2008, On an alternative approach to stress constraints relaxation in topology optimization, Structural and Multidisciplinary Optimization, 36, 125-141

8. Bruggi M., Venini P., 2008, A mixed FEM approach to stress-constrained topology optimization, International Journal for Numerical Methods in Engineering, 73, 1693-1714

9. CAI S.Y., Zhang W.H., 2015, Stress constrained topology optimization with free-form design domains, Computer Methods in Applied Mechanics and Engineering, 289, 267-290 
10. Cai S.Y., Zhang W.H., Zhu J.H., Gao T., 2014, Stress constrained shape and topology optimization with fixed mesh: a B-spline finite cell method combined with level set function, Computer Methods in Applied Mechanics and Engineering, 278, 361-387

11. Cheng K.T., Olhoff N., 1981, An investigation concerning optimal design of solid elastic plates, International Journal of Solids and Structures, 17, 3, 305-323

12. Deaton J.D., Grandhi R.V., 2014, A survey of structural and multidisciplinary continuum topology optimization: post 2000, Structural and Multidisciplinary Optimization, 49, 1, 138

13. Duysinx P., Bendsøe M.P., 1998, Topology optimization of continuum structures with local stress constraints, International Journal for Numerical Methods in Engineering, 43, 8, 1453-1478

14. Emmendoerfer H., Fancello E.A., 2016, Topology optimization with local stress constraint based on level set evolution via reaction-diffusion, Computer Methods in Applied Mechanics and Engineering, 305, 62-88

15. Eschenauer H.A., Olhoff N., 2001, Topology optimization of continuum structures: a review, Applied Mechanics Reviews, 54, 4, 331-390

16. Guo X., Cheng G.D., 2010, Recent development in structural design and optimization, Acta Mechanica Sinica, 26, 6, 807-823

17. Guo X., Zhang W.S., Wang M.Y., Wei P., 2011, Stress-related topology optimization via level set approach, Computer Methods in Applied Mechanics and Engineering, 200, 47, 3439-3452

18. Guo X., Zhang W., Zhong W., 2014a, Doing topology optimization explicitly and geometrically: a new moving morphable components based framework, Journal of Applied Mechanics, 81, 8, 081009

19. Guo X., Zhang W., Zhong W., 2014b, Stress-related topology optimization of continuum structures involving multi-phase materials, Computer Methods in Applied Mechanics and Engineering, 268, 1, 632-655

20. Hemp W.S., 1973, Optimum Structure, Clarendon Press, Oxford, UK

21. Holmberg E., Torstenfelt B., Klarbring A., 2013, Stress constrained topology optimization, Structural and Multidisciplinary Optimization, 48, 33-47

22. Kennedy G.J., Hicken J.E., 2015, Improved constraint-aggregation methods, Computer Methods in Applied Mechanics and Engineering, 289, 332-354

23. Kiyono C.Y., Vatanabe S.L., Silva E.C.N., Reddy J.N., 2016, A new multi-p-norm, formulation approach for stress-based topology optimization design, Composite Structures, 156, 10-19

24. Le C., Norato J., Bruns T., Ha C., Tortorelli D., 2010, Stress-based topology optimization for continua, Structural and Multidisciplinary Optimization, 41, 605-620

25. Michell A.G.M., Melbourne M.C.E., 1904, The limits of economy of material in framestructure, Philosophical Magazine, 8, 6, 589-597

26. París J., Navarrina F., Colominas I., Casteleiro M., 2009, Topology optimization of continuum structures with local and global stress constraints, Structural and Multidisciplinary Optimization, 39, 419-437

27. Parvizian J., Düster A., Rank E., 2012, Topology optimization using the finite cell method, Optimization and Engineering, 13, 1, 57-78

28. Pereira J.T., Fancello E.A., Barcellos C.S., 2004, Topology optimization of continuum structures with material failure constraints, Structural and Multidisciplinary Optimization, 26, 50-66

29. Picelli R., Townsend S., Brampton C., Norato J., Kim H.A., 2018, Stress-based shape and topology optimization with the level set method, Computer Methods in Applied Mechanics and Engineering, 329, 1-23 
30. Prager W., Rozvany G.I.N., 1977, Optimal layout of grillages, Journal of Structural Mechanics, 5, 1, 1-18

31. Rozvany G.I.N., Zhou M., Birker T., 1992, Generalized shape optimization without homogenization, Structural Optimization, 4, 3-4, 250-252

32. Sigmund O., Maute K., 2013, Topology optimization approaches, Structural and Multidisciplinary Optimization, 48, 6, 1031-1055

33. Suresh K., Takalloozadeh M., 2013, Stress-constrained topology optimization: a topological level-set approach, Structural and Multidisciplinary Optimization, 48, 2, 295-309

34. Svanberg K., 1987, The method of moving asymptotes - a new method for structural optimization, International Journal for Numerical Methods in Engineering, 24, 2, 359-373

35. WANG M.Y., Li L., 2013, Shape equilibrium constraint: a strategy for stress-constrained structural topology optimization, Structural and Multidisciplinary Optimization, 47, 3, 335-352

36. Wang M.Y., Wang X.M., Guo D.M., 2003, A level set method for structural topology optimization, Computer Methods in Applied Mechanics and Engineering, 192, 1-2, 227-246

37. Xia Q., Shi T., Liu S., WAng M.Y., 2012, A level set solution to the stress-based structural shape and topology optimization, Computers and Structures, 90-91, 1, 55-64

38. Xie Y.M., Steven G.P., 1993, A simple evolutionary procedure for structural optimization, Computers and Structures, 49, 5, 885-896

39. Zhang W.S., Guo X., Wang M.Y., Wei P., 2013, Optimal topology design of continuum structures with stress concentration alleviation via level set method, International Journal for Numerical Methods in Engineering, 93, 9, 942-959

40. Zhang W.S., Yang W.Y., Zhou J., Guo X., Li D., 2017, Structural topology optimization through explicit boundary evolution, Journal of Applied Mechanics, 84, 1, 011011

41. Zhou K., Li J.F., 2005, Forming Michell truss in three-dimensions by finite element method, Applied Mathematics and Mechanics, English Edition, 26, 3, 381-388

42. Zhou K., Li X., 2006, Topology optimization of structures under multiple load cases using fiber-reinforced composite material model, Computational Mechanics, 38, 2, 163-170

43. Zhou K., Li X., 2011, Topology optimization of truss-like continua with three member model under stress constraints, Structural and Multidisciplinary Optimization, 43, 4, 487-493

44. Zhou M., Rozvany G.I.N., 1991, The COC algorithm, Part II: Topological, geometrical and generalized shape optimization, Computer Methods in Applied Mechanics and Engineering, 89, 309-336 